(2) Isolation. The 55 ribosomal proteins were isolated by CM-cellulose or cellulose phosphate column chromatography followed by Sephadex gel filtration.

(3) Properties. The following chemical, physical and immunological properties of isolated proteins have been studied: ( $a$ ) molecular weights, $(b)$ amino acid compositions, $(c)$ isoelectric points $(d)$ secondary structure, $(e) N$-terminal amino acids, $(f)$ stoicheiometry, $(g)$ peptide 'maps', $(h)$ isolation and analysis of tryptic peptides from 20 proteins, $(i)$ sequence analysis of five proteins and $(j)$ preparation of antibodies against each isolated protein. These studies demonstrate that there are no homologous structures among all proteins except two, namely proteins $\mathrm{L} 7$ and L 12, which differ only at the $N$-terminus.

(4) Topography. To obtain information about the spatial structure of the ribosomal particles the following studies have been done: $(a)$ electron microscopy, (b) X-ray scattering, (c) cross-linking of proteins with bifunctional reagents, $(d)$ stepwise removal of proteins from ribosomes by salt treatment and by enzymic digestion, $(e)$ determination of which proteins are present in the various ribosomal precursors, $(f)$ specific binding of individual proteins to rRNA, $(g)$ construction of an assembly map by reconstitution of $30 \mathrm{~S}$ particles from 16S RNA and individual proteins and $(h)$ determination of the accessibility of proteins in the intact particle for modifying reagents and for antibodies against individual proteins. The combined results from these approaches give some information about the topography of the ribosomal particles.

(5) Function of individual proteins. In spite of the strong structural and functional interactions between the various components in the ribosomal particles, functions could be determined for a number of ribosomal proteins by the following methods: (a) construction of protein-deficient particles by reconstitution with 30S-particle protein mixtures from which single proteins were omitted, $(b)$ chemical modification of individual proteins, $(c)$ studies on the effect of antibodies against individual ribosomal proteins on ribosomal function, $(d)$ addition of single proteins to a 30S-particle protein population that is heterogeneous with respect to some proteins and $(e)$ comparison between the functions of ribosomes from wild-type and from mutants with altered responses to antibiotics.

(6) Mutants with altered ribosomal proteins. (a) In streptomycin-resistant or -dependent mutants protein $\mathrm{S} 12$ is altered. The single amino acid replacements in twelve mutants that have been studied are located at only two amino acid positions of the protein chain. There is a good agreement between protein-chemical and genetic data. (b) Streptomycin-dependence is suppressed by an alteration in protein $\mathrm{S} 4$ or in protein S5. Alterations in protein $\mathrm{S} 4$ can be more extensive than single amino acid replacements, and can lead to differences in molecular weight of protein S4. (c) In mutants resistant to spectinomycin protein $\mathrm{S} 5$ is altered. The single amino acid replacements of the mutants studied so far are located within a very small region of protein $\mathbf{S 5}$. (d) In temperaturesensitive mutants protein $\mathrm{S} 8$ or protein $\mathrm{S} 18$ has been found to be altered. (e) In contrast with the above mutants, which have altered 30S-particle proteins, in mutants resistant to erythromycin a 50S-particle protein, namely protein $\mathrm{L} 4$, is affected. The amino acid replacements are probably clustered. $(f)$ Another $50 \mathrm{~S}$-particle protein, namely protein $\mathrm{L} 22$, is also altered in mutants isolated as erythromycin-resistant mutants.

(7) Naturally occurring $E$. coli strains. No differences have been detected by chromatographic and electrophoretic techniques in any of the 50S-particle proteins from $E$. coli strains K, B, C or M.R.E. 600, but two 30S-particle proteins, namely proteins $\mathrm{S} 5$ and S7, differ among these strains.

(8) Comparison of ribosomes among bacteria. Ribosomal proteins from bacteria belonging to various Families have been compared by electrophoretic and immunological methods. The results show very close relationships among species belonging to the Enterobacteriaceae, whereas other Families, e.g. Bacillaceae, are not so uniform with respect to their ribosomes.

(9) Comparison of ribosomes from bacteria and cell organelles. To test for possible relationships among the class of $70 \mathrm{~S}$ ribosomes, immunological studies have been done with ribosomes from chloroplasts of higher plants, from mitochondria of fungi and from bacteria of various Families. No relationships have been detected among ribosomes from the three groups.

\section{S Ribosomal Proteins}

By Hannah J. Gould, O. H. W. Martini and H. S. W. KING (Department of Biophysics, King's College, University of London, 26-29 Drury Lane, London WC2B 5RL U.K.)

We have applied new analytical methods to solve problems concerning the number and quantities of different ribosomal proteins in eukaryotic ribosomes, ribosomal subunits and messenger ribonucleoprotein particles.

The separation of $80 \mathrm{~S}$ ribosomal proteins has been carried to a higher stage of resolution by the use of two-dimensional polyacrylamide-gel electrophoresis (Martini \& Gould, 1971). The numbers of proteins in ribosomal subunits, 30 in the small subunit and 37 in the large subunit, were calculated from the known average molecular weight and the number of daltons 
of protein, assuming equimolar amounts of all the proteins. The number resolved by two-dimensional polyacrylamide-gel electrophoresis is at least 26 for the small subunit and 36 for the large subunit of rabbit reticulocyte ribosomes. The near-complete resolution of the ribosomal proteins opens up new possibilities for both quantitative and qualitative analyses.

A qualitative survey of the proteins in ribosomes and ribosomal subunits from wide variety of organisms, e.g. birds and mammals, demonstrates a remarkable degree of similarity. Since the position of a protein in the two-dimensional electrophoretogram is dependent on both its net charge and its size, this broad similarity suggests that there is a strong evolutionary pressure to conserve the physicochemical properties of the ribosomal proteins. However, certain proteins appear to be characteristic of the species of origin, e.g. a protein of 45000 molecular weight found in the large subunit derived from the ribosomes of either the liver or reticulocytes of the rabbit.

An additional advantage of the particular method of analysis is the use of sodium dodecyl sulphate in the second dimension, which provides an estimate of the molecular weight of all the separated proteins. Knowledge of the molecular weights plus the relative quantities of the protein is required in order to calculate the stoicheiometry of the proteins in the ribosome. We have recently been investigating methods for determining the relative quantities of individual proteins separated in polyacrylamide-gel slabs, and our progress on this topic will be discussed in some detail.

We have examined the effect of washing ribosomes through solutions of different $\mathrm{KCl}$ concentrations and $\mathrm{KCl} / \mathrm{Mg}^{2+}$ ratios on the removal of proteins, both adsorbed 'contaminants' and 'ribosomal' proteins. At the salt concentrations commonly used to produce active ribosomal subunits it appears that some proteins, which we define as 'ribosomal' proteins on the basis that they appear to be present in amounts of about $1 \mathrm{~mol} / \mathrm{mol}$ of ribosomes in the intact ribosome, may be partially washed off. A few differences between ribosomes in different functional states, e.g. monoribosomes, polyribosomes, native and derived subunits, and ribosomes unfolded with EDTA, have been observed.

EDTA-dissociated polyribosomes and messenger ribonucleoprotein dissociated from ribosomes in the presence of EDTA and purified by zonal centrifugation have also been subjected to two-dimensional polyacrylamide-gel electrophoresis. Three proteins, with molecular weights of approx. 150000,90000 and 60000 , have been found in association with the messenger and one, of molecular weight 37000 , has been found in association with 5S RNA. These results may be compared with those of other workers, who found two proteins, of molecular weights 130000 and 68000 , in association with the messenger (Lebleu et al., 1971) and one, of molecular weight 45000 (Lebleu et al., 1971) or 35000 (Blobel, 1971), in association with 5S RNA in rabbit reticulocytes.

Blobel, G. (1971) Proc. Nat. Acad. Sci. U.S. 68, 1881

Lebleu, B., Marbaix, G., Huez, G., Temmerman, J., Burny, A., \& Chantrenne, H. (1971) Eur. J. Biochem. 19, 265

Martini, O. H. W. \& Gould, H. J. (1971) J. Mol. Biol. 62, 403

\section{The Genes for Ribosomal Ribonucleic Acid}

By PETER J. Ford (Department of Genetics, University of Edinburgh, West Mains Road, Edinburgh EH9 $3 J N$, U.K.)

The coding sequences for rRNA species are reiterated within the genome of all organisms except Mycoplasma (Birnstiel et al., 1971a). There is a general relationship between redundancy of rRNA coding sequences and genome size (Birnstiel et al., 1971a). In prokaryotes the 5S rRNA coding sequences are closely linked to the $16 \mathrm{~S}$ and $23 \mathrm{~S}$ rRNA coding sequences (Jarry \& Rosset, 1970; Smith et al., 1968), unlike eukaryotes, where they are not closely linked to the $18 \mathrm{~S}$ and $28 \mathrm{~S}$ rRNA coding sequences (Brown \& Weber, 1968; Wimber \& Steffensen, 1970; Alloni et al., 1971).

In Xenopus the coding sequences for 5S rRNA are 10000-24000-fold redundant (Birnstiel et al., 1971b; Brown \& Weber, 1968; Brown et al., 1971) and are clustered within the genome (Brown \& Weber, 1968), which has allowed their isolation as a pure DNA satellite (Brown et al., 1971) in a manner similar to the isolation of the $18 \mathrm{~S}$ and $28 \mathrm{~S}$ ribosomal DNA (Birnstiel et al., 1966). The isolation in pure form of a second repetitive DNA coding for an rRNA has allowed the construction of a general scheme for the organization of the repeating unit, consisting of noncoding spacer DNA alternating with the coding DNA sequence, for this class of repetitive DNA in eukaryotes. It remains to be seen whether this applies to tRNA and mRNA coding sequence clusters as well.

The assertion that reiterated coding sequences, such as those for rRNA, are all identical within a species has been debated for several years (Birnstiel et al., 1971a). Nucleic acid molecular hybridization experiments have been used to support this idea (Birnstiel \& Grunstein, 1972) and to deny it (Moore \& McCarthy, 1968). However, the exact significance of the effects of the type and level of base substitutions on the rate of formation (Southern, 1971; Sutton \& McCallum, 1971) and the thermal stability 